\title{
Reticulocyte Corpuscular Hemoglobin Content
}

National Cancer Institute

\section{Source}

National Cancer Institute. Reticulocyte Corpuscular Hemoglobin Content. NCI Thesaurus.

Code C98776.

The determination of the amount of hemoglobin in reticulocytes within a sample. 